\title{
A violência sexual contra a criança e as influências no seu desenvolvimento
}

\author{
The sexual violence against children and influences on their development \\ Violência sexual contra los niños e influencias en su desarrollo
}

Recebido: 20/04/2021 | Revisado: 27/04/2021 |Aceito: 02/05/2021 | Publicado: 16/05/2021

Thalyta Freitas dos Santos Laguna

ORCID: https://orcid.org/0000-0002-4227-0020

Universidade Franciscana, Brasil

E-mail: thalytalaguna@gmail.com

Telma Garcez Leal

ORCID: https://orcid.org/0000-0003-3279-3044

Universidade Franciscana, Brasil

E-mail: telmagarcezleal@hotmail.com

Aline Priscila Subutzki Lemos

ORCID: https://orcid.org/0000-0002-0472-4474 Universidade Franciscana, Brasil

E-mail: asubutzki@hotmail.com

Isadora Ribeiro Meine

ORCID: https://orcid.org/0000-0002-4226-4210 Universidade Franciscana, Brasil E-mail: meineisadora@gmail.com

Henrique Carvalho Rigo

ORCID: https://orcid.org/0000-0001-5638-7062 Universidade Franciscana, Brasil

E-mail: henriquec.rigo@gmail.com

Mariana da Silva Pereira

ORCID: https://orcid.org/0000-0003-1226-5324 Universidade Franciscana, Brasil

E-mail: pereiramariana14@gmail.com

Cristina Saling Kruel

ORCID: https://orcid.org/0000-0003-1996-7708 Universidade Franciscana, Brasil

E-mail: cristinaskruel@gmail.com

\begin{abstract}
Resumo
A violência contra a criança é caracterizada pela presença de abusos de cunho físico, sexual, emocional e psicológico, que podem afetar seu desenvolvimento. Dito isto, este artigo tem por objetivo discorrer, em específico, sobre a violência sexual contra o infante, contemplando desde aspectos relacionados aos agressores, até às consequências implicadas às vítimas, colocando em evidência, desta forma, os fatores que interferem em seu processo de maturação. Por fim, discute sobre questões relacionadas à proteção das vítimas e ao tratamento destas, a fim de evitar a piora dos traumas impressos. Trata-se de um estudo de revisão bibliográfica, para o qual foram feitas pesquisas em artigos, livros e cartilhas que abrangessem o assunto delimitado. Identificamos como resultados que em meio a tantos prejuízos, frutos de eventos estressantes e de natureza ameaçadora, vale ressaltar que a criança vítima de abuso sexual, com ou sem penetração, tem seu processo de maturação e organização cerebral ameaçados. Identificou-se a carência de novos estudos, pesquisas e bibliografias de modo geral que trabalhem o tema, além de poucos métodos de divulgação preventiva. Concluímos, ao final do estudo, que o abuso sexual contra a criança geralmente poderá inscrever na vítima diversas marcas, muitas vezes em decorrência da violência física e psicológica associadas ao evento, uma vez que o sujeito que sofre o abuso, ainda não está preparado - do ponto de vista físico, emocional e psicológico - para o ato sexual.
\end{abstract}

Palavras-chave: Abusos; Agressores; Consequências; Maturação; Proteção.

\begin{abstract}
Violence against children is characterized by the presence of physical, sexual, emotional and psychological abuse, which can affect their development. That said, this article aims to discuss, specifically, sexual violence against the infant, covering aspects related to the aggressors, to the consequences implied for the victims, thus highlighting the factors that interfere in their process of maturation. Finally, it discusses issues related to the protection of victims and their treatment, in order to avoid worsening printed traumas. This is a bibliographic review study, for which research was carried out on articles, books and booklets that covered the delimited subject. We identified as results that in the midst of so many losses, the result of stressful events and of a threatening nature, it is worth mentioning that the child victim of sexual abuse, with or without penetration, has its maturation process and brain organization threatened. The
\end{abstract}


lack of new studies, research and bibliographies in general that deal with the topic was identified, in addition to few methods of preventive dissemination. We concluded, at the end of the study, that sexual abuse against the child will generally be able to inscribe on the victim several marks, often as a result of the physical and psychological violence associated with the event, since the subject who suffers the abuse is not yet prepared - from a physical, emotional and psychological point of view - for the sexual act.

Keywords: Abuses; Offenders; Consequences; Maturation; Protection.

\section{Resumen}

La violencia contra los niños se caracteriza por la presencia de maltrato físico, sexual, emocional y psicológico, que puede afectar su desarrollo. Dicho esto, este artículo tiene como objetivo discutir, específicamente, la violencia sexual contra el infante, abarcando aspectos relacionados con los agresores, con las consecuencias que implica para las víctimas, destacando así los factores que interfieren en su proceso de maduración. Finalmente, se analizan temas relacionados con la protección de las víctimas y su tratamiento, con el fin de evitar el agravamiento de los traumas impresos. Se trata de un estudio de revisión bibliográfica, para lo cual se realizó una investigación sobre artículos, libros y folletos que cubrieron el tema delimitado. Identificamos como resultado que en medio de tantas pérdidas, producto de hechos estresantes y de carácter amenazante, cabe mencionar que el niño víctima de abuso sexual, con o sin penetración, tiene su proceso de maduración y organización cerebral amenazada. Faltaron nuevos estudios, investigaciones y bibliografías en general que aborden el tema, además de pocos métodos de difusión preventiva. Concluimos, al final del estudio, que el abuso sexual contra el niño generalmente podrá inscribir en la víctima varias marcas, muchas veces como resultado de la violencia física y psicológica asociada al evento, ya que el sujeto que sufre el abuso aún no está preparado - desde el punto de vista físico, emocional y psicológico - para el acto sexual.

Palabras clave: Acosos; Agresores; Consecuencias; Maduración; Protección.

\section{Introdução}

A Organização Mundial da Saúde, WHO (2003), classifica a violência contra a criança em: violêcia física, sexual, emocional/psicológica e negligência. Nesse contexto, daremos atenção especial ao abuso de teor sexual, que, ainda segundo apontamentos da WHO (2003), ocorre quando uma criança é submetida a qualquer ato de cunho sexualizado para o qual ela não possui desenvolvimento, maturidade, bem como, sequer deu consentimento.

A violência sexual contra a criança pode ocorrer em meio intrafamiliar, e muitas vezes, pode também ser praticada por pessoas do convívio da família. Este tipo de evento pode gerar sequelas físicas e psicológicas, e, não obstante, impactar no desenvolvimento do infante, visto que, por estarem em fase de neurodesenvolvimento, as crianças são mais propensas a sofrer as consequências desta agressão, que podem ser fisiológicas, cognitivas, psíquicas, sociais, sexuais, dentre outras (Florentino, 2015; Inque \& Ristum, 2008; Platt at al., 2018).

Para o Sistema de Informação de Agravos de Notificação (Sinan), entre 2011 e 2017 foram notificados 1.460 .326 casos de violência interpessoal ou autoprovocada. Isso posto, foram registradas $219.717(15,0 \%)$ notificações contra crianças e 372.014 (25,5\%) contra adolescentes, concentrando 40,5\% de todos os registros (Ministério da Saúde, 2018).

Conforme o Ministério da Saúde (2018), quando avaliadas as características sociodemográficas desse público, viu-se $43.034(74,2 \%)$ eram do sexo feminino e 14.996 (25,8\%) eram do sexo masculino. Deste número, percebeu-se ainda que $51,2 \%$ estavam na faixa etária entre 1 e 5 anos, 45,5\% eram da raça/cor da pele negra, e 3,3\% possuíam alguma deficiência ou transtorno. Os registros foram feitos nas regiões Sudeste (40,4\%), Sul $(21,7 \%)$, Norte $(15,7 \%)$, Nordeste $(12,5 \%)$ e CentroOeste $(9,7 \%)$.

Observando as características da violência sexual, obteve-se que 33,7\% dos casos tiveram caráter de repetição, 69,2\% ocorreram na residência, no meio intrafamiliar, ao passo que 4,6\% ocorreram na escola. Em 81,6\% dos casos o agressor era do sexo masculino e em 37\%, tinham vínculo com a família (Ministério da Saúde, 2018).

Partindo do exposto, este artigo tem por objetivo discorrer sobre a violência sexual contra a criança, contemplando desde aspectos relacionados aos agressores - familiares, parentes, pessoas do convívio - até às consequências implicadas às vítimas, buscando evidenciar, desta forma, os impactos no processo de maturação tanto física, quanto psicológica. Por fïm, discute sobre fatores relacionados à proteção das vítimas e ao tratamento destas, a fim de evitar a piora dos traumas impressos. 


\section{Metodologia}

Nas ciências sociais, a pesquisa qualitativa se preocupa com o universo de significados, crenças e valores, presente nas relações e ações humanas. Dessa maneira, ela visa compreender a realidade das pessoas vivida em âmbito social, vislumbrando, além da experiência e da cotidianidade, as estruturas e instituições como produtos humanos (Deslandes, Neto, Gomes, \& Minayo, 1994). Diante disso, optou-se pela pesquisa bibliográfica em artigos, livros, e cartilhas que abrangessem o assunto delimitado. A principal vantagem desse tipo de pesquisa é a possibilidade de cobrir uma amplitude maior de fenômenos do que aquela que o investigador poderia se pesquisasse de forma direta (Gil, 2002).

Isso posto, este estudo baseou-se na pergunta norteadora "Quais as influências no desenvolvimento da criança que sofre violência sexual?". Os materiais foram pesquisados com base na revisão narrativa da literatura cujo propósito principal é descrever e discutir o desenvolvimento do estado da arte a partir de um ponto de vista teórico (Bardin, 2016).

As palavras chaves utilizadas foram: Abusos; agressores; consequências; maturação; proteção. A coleta de dados se deu por meio de artigos científicos extraídos de revistas nacionais e internacionais nas plataformas Literatura Latino Americana e do Caribe em Ciências da Saúde (Lilacs) e Scientific Eletronic Library Online (Scielo), além dos portais, como Ministério da Educação e da Saúde e Organização Mundial da Saúde. Os resultados e discussão provenientes da pesquisa, foram divididos em "Violência sexual contra a criança: Os agressores" e "Violência sexual contra a criança: Consequências às vítimas".

\section{Resultados e Discussão}

\section{Violência sexual contra a criança: Os agressores}

A literatura abordada neste estudo indica que a maioria dos abusos sexuais contra crianças são praticados por pessoas da família ou próximas a elas e, geralmente, implicam em prejuízos - físicos, psíquicos, sociais, sexuais, entre outros bastante severos no desenvolvimento da criança (Florentino, 2015)

A vista disso, é importante considerar que o impacto na vida das vítimas varia de acordo com as circunstâncias dos fatos e as particularidades de cada indivíduo. Furniss (1993) destaca alguns desses aspectos: a idade da criança, quantidade de vezes em que ocorre o abuso, o grau de violência utilizado no momento da situação, a diferença de idade entre o abusador e o abusado, o vínculo entre eles, a violência psicológica relacionada ao sigilo e outros tipos de ameaças.

Inque e Ristum (2008) referem que a violência psicológica e a física que, frequentemente, acompanham o evento, muitas vezes, imprimem marcas maiores que os da própria violência sexual. Neste âmbito, por exemplo, no abuso sexual intrafamiliar, a criança defronta-se com a possibilidade, após a revelação, da cisão do grupo familiar. Destarte, por não ter ainda maturidade para compreender a gravidade e a dimensão da agressão de que é vítima, opta pelo silêncio, o que, possivelmente, intensificará os efeitos da violência (Inque \& Ristum, 2008; Miranda et al., 2020).

Nesse sentido, Flores e Caminha (1994) destacam que este tipo de violência, representa mais danos para uma criança, pois vem de alguém que é da própria família. Neste caso, o incesto é muito comum e pode vir não apenas da figura masculina, mas também da figura feminina, como por exemplo, entre mãe-filha e mãe-filho (DePanfilis \& Salus, 1992; Matos et al., 2020).

Por conseguinte, podemos observar que na maioria dos casos o abuso vem daquele que tem mais poder sob a vítima, ou seja, de quem detém autoridade dentro de casa ou sob a criança, sendo do mais forte para o mais fraco ou do protetor para o dependente (Gabel, 1997). Isso não enquadra apenas para o pai/padrasto da vítima, mas também para aqueles que a cuidam, como irmãos mais velhos ou avós. Assim sendo, a criança, incapaz de diferenciar as relações, pode se tornar submissa ao seu cuidador, que à priori deveria proporcionar proteção, usa a confiança e a dependência dela para abusá-la sexualmente (Matos et al., 2020). 
Isso posto, famílias incestuosas apresentam relações pessoais assimétricas e hierarquizadas, havendo, então, relações de subordinação. Neste contexto, alguns fatores de risco podem ser observados nesses núcleos familiares: a possibilidade de os pais terem sofrido abuso ou negligência em suas famílias de origem, histórico de uso de drogas, papéis sexuais rígidos, falta de comunicação entre os membros da família, desemprego, mãe passiva e/ou ausente, isolamento social, dificuldades conjugais, pais com transtornos psiquiátricos, dentre outras (Habigzang, Koller, Azevedo, \& Machado, 2005).

Meireles (2013) salienta a vinculação de conceitos culturais de autoritarismo e agressividade em relação às crianças com a tolerância social vinculada às imposições de poder e a exposição delas à violência. Assim, as questões de dominação relacionam-se com gênero, classe social e faixa etária, por exemplo. Com isso, tem-se que a maior parte das ocorrências seja entre pai e filha.

Em paralelo, para Lira et al. (2017), o patriarcado, ao determinar papéis sociais e oportunidades desiguais entre homens e mulheres, incorpora relações de dominação masculina. Dessa forma, são reforçadas a submissão e a perpetuação da violência contra mulheres e meninas. Além disso, Meireles (2013) aponta que o dominador ainda usa a agressividade para manter o poder sobre a vítima.

Araújo (2002) concebe os processos de produção e reprodução da violência na sociedade brasileira como um fenômeno complexo e multicausal. Assim, constata-se ainda a face de banalização e a naturalização da violência, restando às vítimas se adaptarem a ela (Araújo, 2002).

Nesse aspecto, para Furniss (1993), independendemente do grau de cooperação e da vontade de participar do episódio, o que implica, também na dificuldade de noticiar o fato, as crianças que sofrem abuso prolongado desenvolvem fortes sentimentos de culpa e responsabilidade pela experiência. Soma-se a sensação de desamparo e confusão nas interações afetivas da criança, pois o adulto que deveria representar a proteção e confiança torna-se um gerador de perturbações diversas (Furniss, 1993).

Visto que a criança se encontra em fase de desenvolvimento psíquico e físico, as consequências - capazes de aparecer na infância e se estender pela vida adulta ou emergir em outras fases da vida - podem ser múltiplas e vão desde prejuízos ao desenvolvimento orgânico a graves problemas de saúde mental (Florentino, 2015).

Assim, são relacionados sintomas como depressão, sentimento de culpa, medo, comportamento autodestrutivo, isolamento, hostilidade, raiva, estigmatização, autoestima abalada, ansiedade, tendência à revitimização e abuso de substâncias, comportamento sexualizado, queixas somáticas, agressão, rendimento escolar comprometido, transtorno de estresse pós-traumático (TEPT), comportamentos regressivos e ideação suicida (Amazarray \& Koller, 1998; Finkelhor, 1999; Inque \& Ristum, 2008).

\section{Violência sexual contra a criança: Consequências às vítimas}

Em meio a esses prejuízos, frutos de eventos estressantes e de natureza ameaçadora, vale ressaltar que a criança vítima de abuso sexual, com ou sem penetração, tem seu processo de maturação e organização cerebral ameaçados. Boeckel et al. (2016) afirmam que o estresse pode desenvolver uma série de respostas psicobiológicas, uma vez que eventos traumáticos ativam esquemas neurais, particularmente os sistemas nervoso central, periférico, neuroendócrino e imunológico, além do eixo hipotálamo-hipófise-adrenal em especial.

A descarga hormonal desencadeada em momentos de grande estresse, auxilia na mobilização rápida do organismo a ativar mecanismos de defesa ante o objeto estressor. Entretanto, quando em excesso, o cortisol liberado de maneira crônica aumenta a propensão ao desenvolvimento de doenças e pode impactar significativamente no funcionamento cognitivo da criança, havendo possibilidades de apresentar dificuldades de cunho social, educacional e comportamental (Boeckel et al., 2016). 
Não obstante, a vítima de abuso frequentemente sofre do Transtorno de Estresse pós-Traumático (TEPT), psicopatologia anteriormente mencionada e que merece atenção por ser apontada como sendo o transtorno psicológico mais comum dentre os casos de violência sexual contra crianças e adolescentes (Florentino, 2015).

Muito embora a maioria das pessoas que sofreram abuso sexual na infância apresentarem os sintomas de TEPT, não há uma relação de causa-efeito entre a violência e o surgimento de psicopatologias, uma vez que outras também podem - ou não - se manifestar na vítima, como transtorno de déficit de atenção e hiperatividade, transtornos de ansiedade e depressão (Saywitz, Mannarino, Berliner, \& Cohen, 2000).

O diagnóstico desta patologia é feito após a vítima vivenciar ou testemunhar eventos traumáticos e reagir com intenso comportamento de esquiva, pânico, pavor e medo. Isso posto, as crianças que desenvolvem TEPT podem sofrer da chamada reexperiência intrusiva, reencenando os traumas em vários momentos cotidianos, como em brincadeiras, durante o sono, rememorando a experiência através de pesadelos e sonhos traumáticos recorrentes, reconstituição da cena vivida ou lembrança angustiante do momento do abuso (Florentino, 2015).

Ainda, pode frequentemente se esquivar de locais e situações a fim de evitar pensamentos e sentimentos relacionados ao trauma. Essa situação é capaz de ser facilmente observada em crianças vítimas de abuso, quando manifestam interesse diminuído por atividades que antes eram habituais ou apresentam retrocesso em habilidades de desenvolvimento, dificuldades de memória e concentração, hipervigilância, além de respostas de sobressalto demasiadamente exageradas (Borges \& Dell'Aglio, 2008).

Outrossim, ocorre deturpação física, mental, biológica e orgânica, que, segundo Florentino (2015) podem ser: lesões físicas gerais, anais, genitais, doenças sexualmente transmissíveis, hematomas, disfunções sexuais, gestação, além dos já citados problemas de desordem mental, sendo estes últimos muitas vezes causados também pelas lesões de cunho orgânico.

O abuso sexual infantil deve ser visto não só como violação dos direitos infantis, mas também como um problema de que pode afetar a saúde física e mental das vítimas. Em um estudo desenvolvido por Maia e Williams (2005) sobre os fatores de risco e de proteção para o desenvolvimento infantil, foi verificada a importância da capacitação dos profissionais das áreas social, da saúde e da educação para a identificação e encaminhamentos adequados frente à violência contra crianças e adolescentes.

Para tanto, os profissionais e as instituições que constituem a rede de apoio social para crianças e famílias vítimas de abuso sexual encontram-se diante de um desafio: planejar intervenções efetivas que protejam a criança e minimizem os efeitos da violência sofrida (Ferreira \& Schramm, 2000).

Ao encontro disso, Habigzang et al. (2005) ressalta que as consequências do abuso sexual em crianças podem ser aumentadas quando elas não dispõem de uma rede de apoio social e afetiva. Notabiliza-se ainda que, além da ineficiência de políticas públicas e a das práticas de intervenção e de prevenção, o silêncio da vítima e daqueles que protegem o agressor, tal como o de profissionais - que se refugiam defensivamente na ética profissional - atuam como cúmplices (Araújo, 2002).

Atrelado a isso, Meireles (2013) ressalta que a necessidade de sustentar a imagem mistificada da família - como um grupamento natural e perfeito - motiva os seus componentes a se manter em silêncio. Em paralelo, tem-se a ideia de que o meio familiar é um espaço fechado, em que não há interferências externas, no qual tudo pode acontecer. Essa construção social contribui, também, para a manutenção do segredo, permitindo que os abusos se mantenham na família.

Sem dúvida, o tema abuso sexual infantil é um campo de muitas armadilhas a serem descobertas. Para tanto, segundo Furniss (1993), esse tipo de abuso requer um reexame dos procedimentos legais, coloca problemas de proteção à criança, promove e faz progredir aspectos de profissionalização no cuidado adotivo e desafia terapeutas de qualquer orientação e crença a reconsiderarem suas tarefas e técnicas de abordagem.

Carneiro e Cabral (2010) afirmam que é a crença na descoberta, integração e validação da mesma. A importância da 
descoberta e integração de memórias traumáticas, com seus afetos associados, assim como a validação do trauma do paciente são fundamentais.

Assim, para Oliveira (2012) qualquer intervenção precisa considerar os processos internos, a história familiar, os modos de regulação, os recursos, a capacidade de resiliência e a potencialidade de superação para que os padrões de organização e funcionamento anteriores à revelação não retornem, e efetivamente se ofereça um suporte adequado ao enfrentamento do problema. Nesse sentido, a existência de fatores de proteção à criança pode corroborar para o aumento da probabilidade de ruptura do abuso (Meireles, 2013).

Para tanto, defrontar-se com esta problemática exige a efetiva integração de diferentes setores como saúde, segurança, justiça e educação, bem como o envolvimento de toda a sociedade. Além do cuidador, o educador escolar exerce um importante papel na deteç̧ão, no reconhecimento e intervenção nesses casos de violência, pois, no sistema escolar brasileiro, a criança permanece na escola grande parte de seu dia (Inque \& Ristum, 2008).

Portanto, desde o debate ampliado com toda a sociedade, à construção de políticas públicas e investimentos, são fundamentais na prevenção dos agravos, riscos e tratamento integrado às vítimas ("Violência infantil: uma análise das notificações compulsórias, Brasil 2011”, 2015) (Rates, Melo, Mascarenhas, \& Malta, 2015).

\section{Considerações Finais}

O objetivo deste artigo foi discorrer sobre a violência sexual contra a criança, buscando evidenciar os impactos no processo de seu desenvolvimento. Isso posto, é importante salientar que durante a pesquisa, os materiais encontrados, em sua maioria, voltavam-se para as mesmas fontes, dificultando a busca de novos dados e informações que remetessem ao tema. Isso evidencia que, apesar de ser um assunto relevante, sobretudo, atual, há um avanço lento nas pesquisas sobre o abuso sexual infantil, mesmo havendo muitos estudos sobre o assunto. Neste sentido, por ser um tema de grande importância, sugere-se pesquisas atualizadas voltadas especialmente aos impactos psicológicos nas crianças e adolescentes vítimas de abuso sexual.

Todavia, tomando como base as bibliografias consultadas, ao final do estudo pudemos inferir que o abuso sexual contra a criança geralmente pode inscrever na vítima diversas marcas, muitas vezes relacionadas a violência física e psicológica associadas ao evento. Sendo o sujeito que sofre o abuso, uma criança, o seu desenvolvimento pode ficar bastante comprometido, visto que a ocorrência do fato se dá com quem ainda não está preparado - do ponto de vista físico, emocional e psicológico - para o ato sexual.

Ressalta-se que a dinâmica dos efeitos do abuso sexual e sua extensão é subjetiva, porém a criança abusada é uma vítima potencial para o desencadeamento das diversas consequências citadas neste artigo. Ante ao exposto, é necessário, também, direcionar cada vez mais as propostas de intervenções diretamente ao público-alvo, ou seja, as próprias crianças.

Nessa perspectiva, é importante atentar ao fato de que, a informação, aliada ao acolhimento, especialmente nas creches, escolas e afins, deve ser abordada conforme a idade e cultura das crianças almejando a sua proteção, tanto preventivamente, quanto no sentido de minimizar os danos dessa violência. Exemplo disso é o livro infantil Pipo e Fifi, de autoria de Caroline Arcari (Editora Caqui), o qual explica às crianças, a partir dos três anos de idade, conceitos básicos sobre o corpo, sentimentos, convivência e trocas afetivas, ensinando a diferenciar toques de amor de toques abusivos.

Destarte, instrumentos desse tipo podem contribuir disseminando formas de conhecimento, cuidado e proteção tão essenciais aos pequenos e que podem fazer muita diferença em suas vidas.

\section{Referências}

Amazarray, M. R., \& Koller, S. H. (1998). Alguns aspectos observados no desenvolvimento de crianças vítimas de abuso sexual. Psicologia: Reflexão e Crítica, 11(3). https://www.redalyc.org/pdf/188/18811314.pdf 
Araújo, M. de F. (2002). Violência e abuso sexual na família. Psicologia em Estudo, 7(2), 3-11. https://doi.org/10.1590/S1413-73722002000200002

Bardin L. Análise de conteúdo. Edições 70; 2016

Boeckel, M. G., Adriana, W., Kluwe-Schiavon, B., Camargo, J., \& Grassi-Oliveira, R. (2016). Família, estresse e aspectos neurocognitivos: um modelo desenvolvimental. In Neuropsicologia do desenvolvimento: Infância e adolescência (p. 253-264). Artmed.

Borges, J. L., \& Dell'Aglio, D. D. (2008). Relações entre abuso sexual na infância, transtorno de estresse pós-traumático (TEPT) e prejuízos cognitivos. Psicologia em Estudo, 13(2), 371-379. https://doi.org/10.1590/S1413-73722008000200020

Carneiro, S. L. M. A., \& Cabral, M. A. A. (2010). "O silêncio dos inocentes” - abuso sexual intrafamiliar na infância. Revista EPOS, 1(1). Recuperado de http://pepsic.bvsalud.org/scielo.php?script=sci_arttext\&pid=S2178-700X2010000100005

DePanfilis, D., \& Salus, M. (1992). A coordinated response to child abuse and neglect: A basic manual. Washing ton, DC: National Center on Child Abuse and Neglect.

Deslandes, S. F., Neto, O. C., Gomes, R., \& Minayo, M. C. de S. (1994). Pesquisa social: teoria, método e criatividade. Vozes.

Ferreira, A. L., \& Schramm, F. R. (2000). Implicações éticas da violência doméstica contra a criança para profissionais de saúde. Revista de Saúde Pública, 34(6), 659-665. https://doi.org/10.1590/S0034-89102000000600016

Finkelhor, D. (1999). Child sexual abuse. challenges facing child protection and mental health professionals.

Florentino, B. R. B. (2015). As possíveis consequências do abuso sexual praticado contra crianças e adolescentes. Fractal: Revista de Psicologia, 27(2), 139144. https://doi.org/10.1590/1984-0292/805

Flores, R. Z., \& Caminha, R. M. (1994). Violência sexual contra crianças e adolescentes: algumas sugestões para facilitar o diagnóstico correto. Revista de Psiquiatria do RS, 16(2), 158-167.

Furniss, T. (1993). Abuso Sexual da Criança: uma abordagem multidisciplinar. Artes Médicas.

Gabel, M. (1997). Crianças vítimas de abuso sexual (2a ed). Summus Editorial.

Gil, A. C. (2002). Como elaborar projetos de pesquisa (4a ed). Atlas.

Habigzang, L. F., Koller, S. H., Azevedo, G. A., \& Machado, P. X. (2005). Abuso sexual infantil e dinâmica familiar: aspectos observados em processos jurídicos. Psicologia: Teoria e Pesquisa, 21(3), 341-348. http://www.scielo.br/pdf/\%0D/ptp/v21n3/a11v21n3.pdf

Inque, S. R. V., \& Ristum, M. (2008). Violência sexual: caracterização e análise de casos revelados na escola. Estudos de Psicologia, 25(1), 11-21. http://www.scielo.br/pdf/estpsi/v25n1/a02v25n1

Lira, M. O. de S. C. e, Nitschke, R. G., Rodrigues, A. D., Rodrigues, V. P., Couto, T. M., \& Diniz, N. M. F. (2017). Sobrevivendo ao abuso sexual no cotidiano familiar: formas de resistência utilizadas por crianças e adolescentes. Texto Contexto Enfermagem, 26(2). https://doi.org/10.1590/010407072017000050016

Maia, J. M. D., \& Williams, L. C. de A. (2005). Fatores de risco e fatores de proteção ao desenvolvimento infantil: uma revisão da área. Temas em Psicologia, 13(2), 91-103. https://www.redalyc.org/pdf/5137/513751425002.pdf

Matos, K. J. N., Pereira, A. C. S., Lourinho, L. A., Pinto, F. J. M., Gondim, R. S., Matos, G. D. N. (2020). Research, Society and Development, 9(9), e954997952, http://dx.doi.org/10.33448/rsd-v9i9.7952

Meireles, L. de M. (2013). Fatores de risco e fatores de proteção no contexto sociofamiliar das crianças vítimas de abuso sexual atendidas pelo poder judiciário (Universidade Católica de Brasília). https://repositorio.ucb.br/jspui/bitstream/123456789/6694/1/Laura de Melo Meireles.pdf

Ministério da Saúde. (2018). Análise epidemiológica da violência sexual contra crianças e adolescentes no Brasil, 2011 a 2017. Boletim Epidemiológico, 49(27), 1-17. https://portalarquivos2.saude.gov.br/images/pdf/2018/junho/25/2018-024.pdf

Miranda, M. H. H., Fernandes, F. E. C. V., Melo, R. A de, \& Meireles, R. C. (2020). Violência sexual contra crianças e adolescentes: uma análise da prevalência e fatores associados. Revista da Escola de Enfermagem da USP, 54, e03633. https://doi.org/10.1590/s1980-220x2019013303633

Oliveira, A. C. de. (2012). Abuso sexual intrafamiliar de crianças e a família como totalidade. O Social em Questão, 15(28), 233-262. http://osocialemquestao.ser.puc-rio.br/media/12artigo.pdf

Platt, V. B., Back, I. C., Hauschild, D. B., Guedert, J. M. (2018). Violência sexual contra crianças: autores, vítimas e consequências. Ciênc. saúde colet. 23 (4) https://doi.org/10.1590/1413-81232018234.11362016

Rates, S. M. M., Melo, E. M. de, Mascarenhas, M. D. M., \& Malta, D. C. (2015). Violência infantil: uma análise das notificações compulsórias, Brasil 2011. Ciência \& Saúde Coletiva, 20(3), 655-665. https://doi.org/10.1590/1413-81232015203.15242014

Saywitz, K. J., Mannarino, A. P., Berliner, L., \& Cohen, J. A. (2000). Treatment of sexually abused children and adolescents. American Psychologist, 55(9), 1040-1049. https://doi.org/10.1037/0003-066X.55.9.1040

Violência infantil: uma análise das notificações compulsórias, Brasil 2011. (2015). Ciência \& Saúde Coletiva, 20(3), 655-665. https://doi.org/10.1590/141381232015203.15242014

WHO. (2003). Child sexual abuse. In Guidelines for medico-legal care for victims of sexual violence (p. 75-93). https://apps.who.int/iris/bitstream/handle/10665/42788/924154628X.pdf;jsessionid=59E94875C18C7B1AC7DF7085BE6A623B ?sequence=1 